\title{
Tuberculosis miliar paucibacilar secundaria al manejo con etanercept en artritis reumatoide. Reporte de caso
}

\author{
Paucibacilar miliar tuberculosis secondary to management \\ with etanercept in rheumatoid arthritis. A case report
}

Santiago Romero ${ }^{1}$, Maria Daniela Polania ${ }^{2}$, Carolina Prieto ${ }^{3}$, Andreina Zannin Ferrero $^{3}$, Paola Gabriela Zuleta Quintero ${ }^{3}$, Luis Gustavo Celis ${ }^{4}$

\begin{abstract}
Resumen
Los agentes biológicos son medicamentos innovadores que previenen la progresión del daño articular en pacientes con diversas patologías autoinmunes, teniendo impacto sobre su funcionalidad y calidad de vida. El Etanercept (ETN) es el primer agente anti tumoral (anti-TNF) aprobado para el tratamiento de la artritis reumatoide. A pesar de los beneficios evidentes con el uso de medicamentos biológicos, se ha observado que su uso está relacionado con múltiples efectos adversos, siendo los más frecuentes las infecciones oportunistas y granulomatosas, así como también el desarrollo de la tuberculosis. Existen ensayos clínicos que parecen indicar que el riesgo de desarrollar tuberculosis es menor cuando se usa etanercept vs infliximab (IFX) o adalimumab (ADA), en aquellos pacientes con artritis reumatoide, en comparación con los agentes biológicos anti-TNF.

El Factor de Necrosis Tumoral (TNF) es una citoquina importante en la respuesta inflamatoria en el proceso de enfermedades autoinmunes y en el desarrollo de infecciones, por lo cual, los agentes anti-TNF son recomendados en pacientes con artritis reumatoide clasificada de moderada a grave. Sin embargo, tienen un alto riesgo de desarrollar enfermedades inmunosupresoras como la tuberculosis activa. Además, existe mayor riesgo de desarrollar tuberculosis activa con anticuerpos monoclonales frente a las proteínas de fusión.

Se presenta el caso clínico de una mujer con antecedente de importancia de artritis reumatoide en manejo con etanercept y metotrexato, con diagnóstico de tuberculosis miliar paucibacilar secundaria al uso de inhibidores de TNF-alfa.
\end{abstract}

Palabras clave: Tuberculosis miliar; etanercept; artritis reumatoide; factor de necrosis tumoral; TNF; inmunosupresión.

\footnotetext{
${ }^{1}$ Médico, Residente de Medicina Interna. Facultad de Medicina Universidad El Bosque. Bogotá, Colombia.

${ }^{2}$ Médico Facultad de Medicina Universidad de la Sabana, Especialista en Epidemiología. Universidad del Rosario- CES. Bogotá, Colombia.

${ }^{3}$ Médico, Facultad de Medicina. Universidad de la Sabana. Bogotá, Colombia.

${ }^{4}$ Facultad de Medicina, Universidad de la Sabana. Magíster en Biología con énfasis en Genética Humana, Universidad de Los Andes. Bogotá, Colombia.
}

\author{
Autor de Correspondencia \\ Carolina Prieto Soler \\ Correo electrónico: \\ carolinaprso@gmail.com
}

Recibido: 24/09/2020

Aceptado: 20/12/2020 


\begin{abstract}
Biological agents are innovative medicines that prevent the progression of joint damage in patients with various autoinmune diseases, impacting on their functionality and quality of life. Etanercept (ETN) is the first anti tumor necrosis factor agent (anti - TNF) approved for the treatment of rheumatoid arthritis. Despite the obvious benefits of using biological drugs in the treatment of rheumatoid arthritis, it has been observed that their use is associated with the develpoment of multiple adverse effects, such as an increase in the number of infections, like the opportunistic and granulomatous infections and the development of tuberculosis. There are clinical trials that seem to indicate that the risk of developing tuberculosis is lower when using etanercept (ETN) vs infliximab (IFX), Adalimumab (ADA), in patients with rheumatoid arthritis compared to anti - TNF biological agents.

TNF is an important cytokine in the inflammatory response, in the process of autoinmune diseases and in the development of infections, anti - TNF agents are recommended in patients with moderate to severe rheumatoid arthritis, however, they are at high risk of developing immunosuppressive diseases such as active tuberculosis, there is an increases risk of developing active tuberculosis with monoclonal antibodies to fusión proteins. The clinical case of a woman with a history of significant rheumatoid arthiris in management with etanercept and methotrexate is presented. She was diagnosed with paucibacilary miliary tuberculosis secondary to the use of TNF - alpha.
\end{abstract}

Keywords: Miliary tuberculosis; etanercept; rheumatoid arthritis; tumor necrosis factor; TNF; immunosuppression.

\section{Introducción}

Los agentes biológicos son medicamentos innovadores que previenen la progresión del daño articular en pacientes con diversas patologías autoinmunes, teniendo impacto sobre su funcionalidad y calidad de vida $(1,2)$. Se ha evidenciado que el Factor de Necrosis Tumoral (TNF) tiene un papel activo en la fisiopatología de múltiples enfermedades, entre ellas la artritis reumatoide. Por lo cual, actualmente este se ha convertido en uno de los puntos claves del tratamiento de esta enfermedad $(1,2)$. Entre los agentes biológicos cuya diana terapeútica es la inhibición del TNF, se encuentran los anticuerpos monoclonales (adalimumab, certolizumab, infliximab, adalimumab) y las proteínas de fusión (etanercept, abatacept) $(1,2)$.

A pesar de los beneficios evidentes con el uso de medicamentos biológicos en el tratamiento de la artritis reumatoide, se ha observado que su uso está relacionado con el desarrollo de múltiples efectos adversos debido al incremento del número de infecciones, siendo las más frecuentes las infecciones oportunistas y granulomatosas, así como también el desarrollo de la tuberculosis (TB), principalmente en pacientes en manejo con medicamentos que tienen efectos antagonistas del factor de necrosis tumoral alfa (FNT $\alpha)(1,2)$.

Según los registros de uso de biológicos de la So- ciedad Británica de Reumatología, el tiempo promedio de diagnóstico de tuberculosis activa en pacientes con uso de antagonistas del factor de necrosis tumoral alfa $(\mathrm{FNT} \alpha)$ varía entre 5.5-18.5 meses. Además, estos registros evidencian que la menor tasa de incidencia de esta infección se encuentra en los pacientes que reciben etanercept (1).

A continuación se presenta el caso de una paciente femenina de 63 años con artritis reumatoide, en manejo con etanercept, en quien se realiza diagnóstico de tuberculosis miliar activa a los tres años de tratamiento con terapia biológica, con el fin de describir la relación del desarrollo de tuberculosis activa durante el tratamiento con fármacos anti-TNF. Se destaca la importancia del cribado de infección latente tuberculosa previo al inicio del tratamiento y la realización de un seguimiento clínico, para detectar signos de tuberculosis con cuadros atípicos y/o diseminados.

Tras el diligenciamiento del consentimiento informado y luego de la revisión de la historia clínica completa y de los exámenes de laboratorio de la paciente, se realizó una búsqueda bibliográfica en bases de datos como Science Direct, Clinicalkey, PubMed, y OMIM, acerca de la tuberculosis miliar paucibacilar secundaria al manejo con etanercept, en pacientes que presentan artritis reumatoide como antecedente de importancia. 


\section{Reporte de Caso}

Paciente femenina de 63 años de edad, natural y procedente de Bogotá, quien consulta al servicio de urgencias de la Clínica Occidente por presentar un cuadro clínico de 20 días de evolución consistente en disuria, polaquiuria y urgencia miccional. Adicionalmente, la paciente refiere tres semanas de presencia de tos no productiva, diaforesis nocturna, pérdida de peso y fiebre no cuantificada. Presenta como antecedente patológico de importancia artritis reumatoide, diagnosticada en el año 2009 dada la clínica de poliartralgias y artritis de articulaciones pequeñas periféricas (principalmente interfalángicas proximales), asociada a elevación de reactantes de fase aguda, factor reumatoideo positivo $(29 \mathrm{UI} / \mathrm{ml}$ ) y anticuerpo anticitrulina positivo (133.2 U/ml). Recibió manejo con prednisona y metotrexate vía oral y posteriormente en el año 2016 al presentar pobre respuesta al tratamiento, se cambia manejo farmacológico con etanercept semanal y metotrexato subcutáneo.

Se realiza uroanálisis con resultados sugestivos de infección, por lo cual se inicia manejo antibiótico intrahospitalario. El urocultivo es negativo por lo que se suspende el manejo antibiótico, sin embargo, subsecuentemente durante la hospitalización, se evidencian saturaciones de oxígeno bajas, por lo que se procede a solicitar una radiografía de tórax, en la cual se obser- van lesiones micronodulares con distribución aleatoria en ambos campos pulmonares asociado a atelectasias planas en lóbulo medio del pulmón derecho (Figura 1).

Dados los hallazgos, se procedió a realizar TAC de tórax, confirmando los resultados previos (Figura 2).

Se realiza prueba de baciloscopia, la cual es negativa; hemograma sin leucocitosis ni neutrofilia, sin anemia y sin alteraciones en el conteo plaquetario.

La paciente es valorada por neumología; se considera que según su contexto clínico es factible una tuberculosis miliar paucibacilar, secundaria al uso de anti-TNF. Se construye el diagnóstico bajo antecedentes, síntomas respiratorios de larga data y criterios radiológicos. En conjunto entre neumología e infectología se decide no se realizar fibrobroncoscopia, ya que en la mitad de los casos el resultado de este procedimiento es negativo.

Dado lo anterior, se inicia tratamiento con rifampicina, isoniazida, etambutol y pirazinamida. Al noveno día de hospitalización, la paciente presenta mejoría notable de síntomas respiratorios, sin actividad de la enfermedad de base, por lo cual se considera alta la posibilidad de presentar infección por tuberculosis. Se da egreso con indicación de manejo, seguimiento de la artritis reumatoide y tuberculosis por parte de reumatología y neumología de manera ambulatoria.

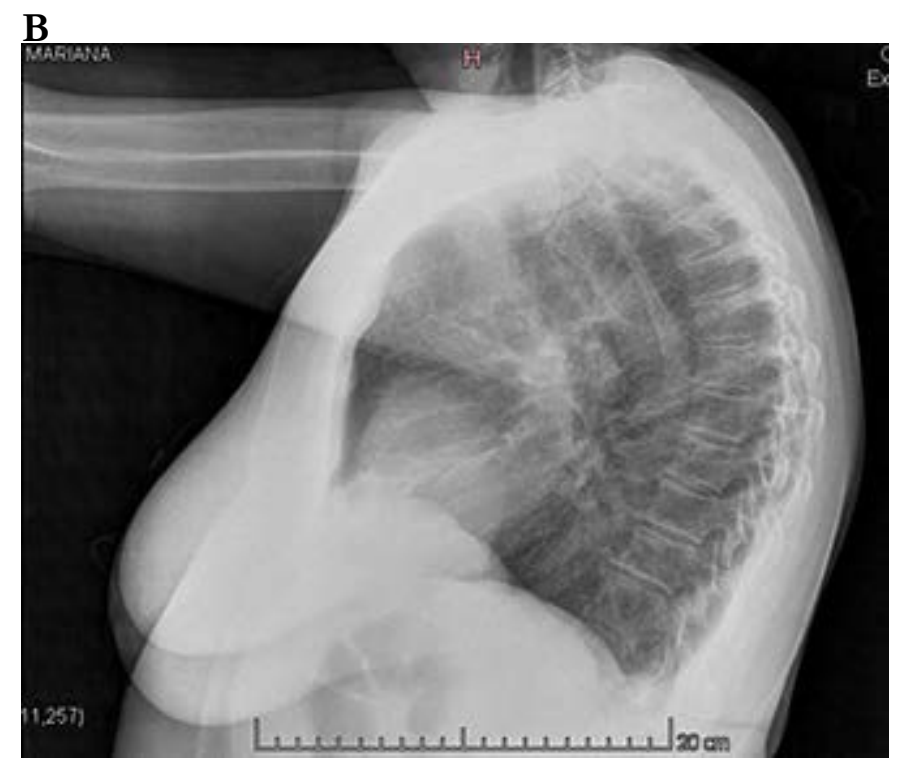

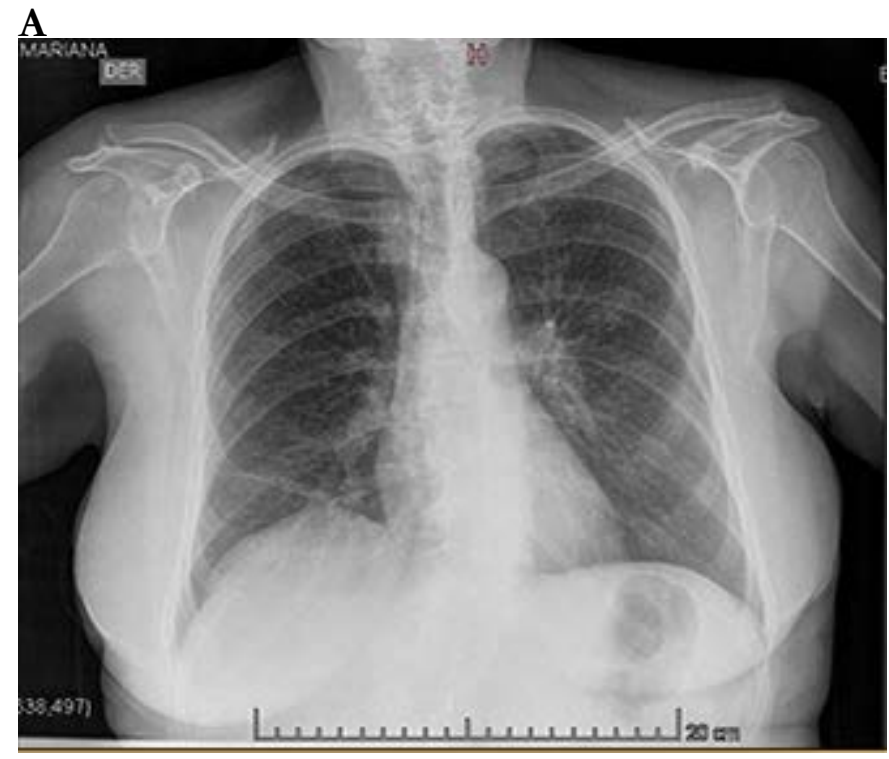

Figura 1. Radiografía de tórax. A. Proyección anteroposterior. B. Proyección lateral. Se observan lesiones micronodulares con distribución aleatoria en ambos campos pulmonares asociado a atelectasias planas en lóbulo medio del pulmón derecho. 


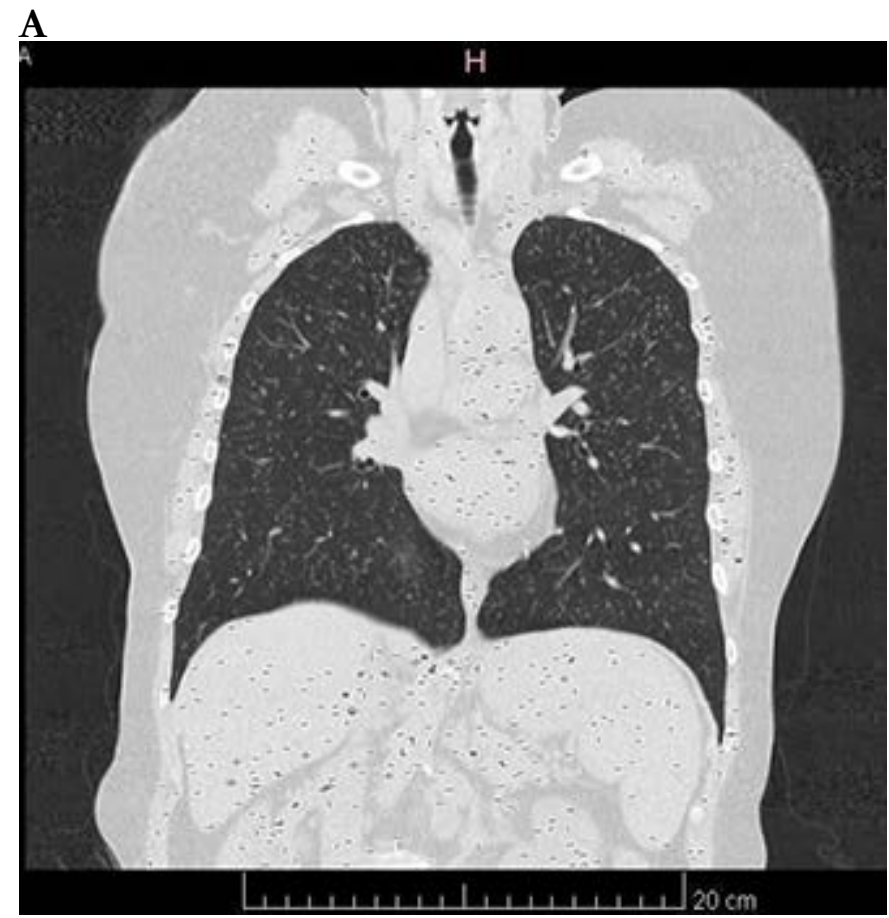

B

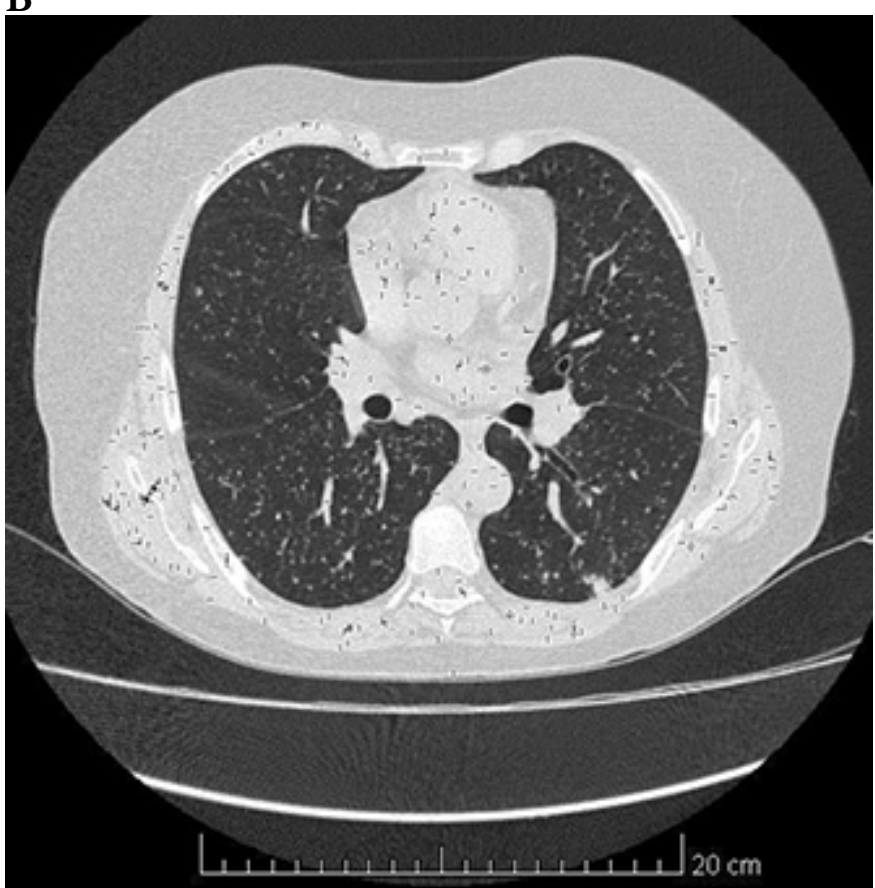

Figura 2. TAC de tórax. A. Corte coronal. B. Corte transversal. Se observan múltiples hiperdensidades con patrón de nódulo y micronódulo de distribución aleatoria compatibles con diseminación hematógena.

\section{Discusión}

El Mycobacterium tuberculosis es el agente causal del desarrollo de la tuberculosis, una enfermedad infecciosa en donde el ser humano sirve como reservorio natural de la bacteria. Uno de los principales factores de riesgo para desarrollar esta enfermedad es el uso de inhibidores de TNF-alfa y su receptor (3).

Actualmente un tercio de la población mundial está infectada con M. tuberculosis y el $10 \%$ de los infectados desarrolla la enfermedad en algún momento de su vida. La mitad de ellos durante los 18 primeros meses después de la infección inicial y el otro $5 \%$ en algún momento posterior. La Organización Mundial de la Salud (OMS) reporta que en 2018 se enfermaron de TB 10 millones de personas, de las cuales 1.5 millones fallecieron a causa de la enfermedad (4). En Colombia la TB es considerada como un evento de interés en salud pública, ya que se ha posicionado como una de las enfermedades transmisibles que aporta un número importante de casos al sistema de vigilancia (5).

Los medicamentos biológicos son aquellos cuyo principio activo se produce o se extrae a partir de una fuente biológica como tejidos, fluidos animales, hu- manos o hemoderivados, entre otros. Medicamentos como los antagonistas del factor de necrosis tumoral alfa, han evidenciado que pueden ser una excelente alternativa en el tratamiento de enfermedades inflamatorias inmunes cuando no hay respuesta a los medicamentos inmunomoduladores convencionales (6). Por lo tanto, se ha observado que su uso se asocia a un riesgo mayor de desarrollar infecciones por agentes patógenos intracelulares especialmente como Mycobacterium tuberculosis. La incidencia de tuberculosis en pacientes bajo tratamiento anti-TNF alfa varía según estudios y áreas geográficas. La Food and Drug Administration (FDA) ha llegado a calcular tasas mundiales de tuberculosis de 20.7 por 100.000 habitantes bajo tratamiento con etanercept y 47.6 por 100.000 habitantes, quienes recibían infliximab (7).

Los mecanismos de acción de estos medicamentos son variados y existen diferentes blancos terapéuticos, tales como el factor de necrosis tumoral $\alpha(\mathrm{TNF}-\alpha)$, la interleucina 1 (IL 1), la interleucina 6 (IL 6), así como los linfocitos B y T (6).

$\mathrm{El} \mathrm{TNF}$ es una citoquina proinflamatoria con un papel importante en la patogénesis de enfermedades inflamatorias o autoinmunes como la artritis reumatoi- 
de, la enfermedad inflamatoria intestinal, la espondilitis anquilosante o la psoriasis (1). Por tal motivo es un blanco terapéutico importante para evitar el desarrollo crónico de estas enfermedades y es aquí donde los inhibidores de citocinas proinflamatorias más conocidos como los anti-TNF, han tomado un papel vital en los últimos años para la base terapéutica de los pacientes con este tipo de enfermedades que no han respondido a una terapia estándar, frente a inmunosupresores no específicos (1). Actualmente existen cinco agentes anti-TNF aprobados para su uso clínico: el etanercept, el infliximab (IFX), adalimumab (ADA), golimumab y certolizumab, que son anticuerpos monoclonales (1).

De estos medicamentos biológicos, el etanercept, es una proteína obtenida mediante la fusión de una porción del receptor soluble del TNF llamado p 75 con un fragmento de inmunoglobulina $G$ humana. Dado el mecanismo de acción que presenta, se facilita la progresión de infección tuberculosa latente (LTBI) a tuberculosis activa, al desarrollo de infecciones oportunistas o la reactivación de las mismas (8). Así, el riesgo de desarrollar estas patologías podría incrementarse en asociación con el uso de inhibidores de TNF (2).

El uso de etanercept está indicado para aquellos pacientes con artritis reumatoide en combinación con un inhibidor competitivo de la dihidrofolato-reductasa como el metotrexato (9). Cuando la terapia antirreumática ha sido la inadecuada, esta combinación de medicamentos es la indicada para pacientes con artritis reumatoide activa que se clasifique como moderada o grave, sin embargo, también se puede administrar etanercept como monoterapia en caso de que el metotrexato no sea apropiado o exista intolerancia al medicamento (2).

Existen ensayos clínicos que parecen indicar que el riesgo de desarrollar tuberculosis es menor cuando se usa etanercept vs infliximab (IFX), adalimumab (ADA) en pacientes con artritis reumatoide en comparación con agentes biológicos anti-TNF (10). El riesgo de reactivación de TB latente es mayor en la terapia con IFX y ADA que con etanercept, siendo de 136$144 / 100.000$ versus $39 / 100.000$, respectivamente, en regiones con una incidencia de TB de 13,2/100.000 habitantes/año (10). Además, según la Sociedad Británica de Reumatología, la incidencia por año de tuberculosis con el uso de anticuerpos monoclonales es más frecuente con adalimumab, seguido de infliximab, en comparación con el uso de etanercept con una incidencia de 39/100.000 habitantes en un período de
13.4 meses (11).

Existen múltiples formas clínicas de tuberculosis que se pueden desarrollar durante el tratamiento anti-TNF, siendo las más frecuentes las extra pulmonares o incluso diseminadas y que pueden llegar a convertirse en factores potencialmente mortales $(12,13)$.

El caso clínico descrito previamente, se trata de una paciente con varios factores de riesgo para desarrollar una tuberculosis activa, pues está cursando con una artritis reumatoide en tratamiento con etanercept, proveniente de zona endémica y no recibió quimioprofilaxis previa a tratamiento con anti-TNF. Sin embargo, no fue del todo sencillo llegar al diagnóstico definitivo ya que se requirió de un manejo multidisciplinario en conjunto con neumología para llegar al diagnóstico de tuberculosis miliar. Los síntomas son muy inespecíficos, por lo cual en primera instancia en el caso presentado, fue muy difícil realizar un diagnóstico inicial, ya que sólo manifestó como síntoma cardinal astenia, adinamia y disnea $(12,13)$.

Los hallazgos iniciales en una radiografía de tórax en pacientes con esta patología pueden no ser evidentes inclusive hasta el final de dicha enfermedad (12). Otra alternativa más sensible para estos casos es la tomografía de alta resolución (TACAR) que evidencia nódulos similares, aunque distribuidos aleatoriamente (12). Por otro lado, el uso de la Resonancia Magnética (MRI) y la ecografía, son útiles en aquellos casos en que se diagnostique una tuberculosis miliar, con el fin de identificar la localización comprometida (12). En varios países, incluyendo Colombia, se han emitido recomendaciones institucionales, de manera que antes de iniciar una terapia con anti-TNF, se realice una historia clínica detallada y se indague sobre antecedentes personales de tuberculosis o un posible contacto previo, fecha de vacunación con BCG, resultados de pruebas de estándar con tuberculina cutánea (PPD) y la medición de producción de interferón gamma (Interferón $\gamma$ Relase Assays, IGRA) que comúnmente se usa en el país (9).

En los casos en que la PPD sea mayor de $5 \mathrm{~mm}$ se recomienda la toma de baciloscopia (BK) por esputo seriado por tres días seguidos (10). El consenso final de la CDC recomienda el tratamiento para tuberculosis a todos los pacientes que planean tomar un inhibidor de TNF, con un resultado PPD positivo (induración mayor o igual a $5 \mathrm{~mm}$ ), un resultado IGRA positivo o en el caso que presenten una PPD o IGRA negativo, pero con evidencia radiológica y/o epidemiológica 
con exposición previa (9).

Hasta el 2018 en Colombia existía un total de diez casos diagnosticados con tuberculosis asociada al uso de medicamentos anti-TNF alfa en diferentes ciudades. En seis de estos, el tratamiento anti-TNF alfa se formuló para el manejo de artritis reumatoide, en tres se formuló por espondilitis anquilosante y en uno para síndrome de Sjogren, (7) más el caso que actualmente se reporta.

Para concluir, los agentes anti-TNF generan gran impacto sobre la progresión de la artritis reumatoide, mejorando la calidad de vida de los pacientes, sin embargo, conllevan un aumento del riesgo de enfermedades oportunistas como la tuberculosis activa, frente al de la población en general. Por ende, la necesidad de descartar la tuberculosis latente en aquellos individuos en los que se va a iniciar el tratamiento con agentes anti-TNF alfa es muy importante. Se estima que entre 2000 y 2018 se salvaron 58 millones de vidas gracias al diagnóstico y tratamiento de la tuberculosis, por lo que poner fin a esta epidemia para el 2030 es una de las metas relacionadas con la salud de los Objetivos de Desarrollo Sostenible para la OMS.

\section{Referencias}

1. Dixon WG, Hyrich KL, Watson KD, Lunt M, Galloway J, Ustianowski A, Symmons DPM. Drug-specific risk of tuberculosis in patients with rheumatoid arthritis treated with anti-TNF therapy: Results from the British Society for Rheumatology Biologics Register (BSRBR). Ann Rheum Dis. 2010;69(3), 522-528. doi: 10.1136/ ard.2009.118935

2. Cantini F, Niccol, L, Goletti D. Adalimumab, etanercept, infliximab, and the risk of tuberculosis: Data from clinical trials, national registries, and postmarketing surveillance. J. Rheumatol. 2014;41(SUPPL.91):47-55. doi: 10.3899/jrheum.140102.

3. Shaaban HS, Bishop SL., Menon L, Slim, J. Mycobacterium chelonae infection of the parotid gland. J Glob Infect Dis. 2012;4(1):79-81. doi: 10.4103/0974-777X.93767.

4. Organización Mundial de la Salud. Informe mundial sobre la tuberculosis 2019. Ginebra: OMS, 2019. Disponible en: https://www.who.int/tb/ publications/global_report/gtbr2019_ExecutiveSummary_sp.pdf
5. Instituto Nacional de Salud. Una mirada hacia el comportamiento de la tuberculosis en Colombia. (Semanas epidemiológicas 1-36). BES Boletín Epidemiológico Semanal. Bogotá, D.C.: INS, 2018. Disponible en: https://bit.ly/2WU08YY

6. Palladino MA, Bahjat FR, Theodorakis EA, Moldawer LL. Anti-TNF- $\alpha$ therapies: The next generation. Nat. Rev. Drug Discov. 2003;2(9):736746. doi: $10.1038 / \mathrm{nrd} 1175$.

7. Galvis L, Sánchez ÁY, Jurado LF, Murcia MI (2018). Tuberculosis asociada a antagonistas del factor de necrosis tumoral alfa, presentación de un caso y análisis de los casos reportados en Colombia. Biomedica. 2018;38(1):7-16. doi: 10.7705/biomedica.v38i0.3273

8. Kim J, Toda T, Watanabe K, Shibuya S, Ozawa Y, Izuo N, Cho S, Seo DB, Yokote K, Shimizu T. Syringaresinol Reverses Age-Related Skin Atrophy by Suppressing FoxO3a-Mediated Matrix Metalloproteinase-2 Activation in Copper/Zinc Superoxide Dismutase-Deficient Mice. J. Invest. Dermatol. 2019;139(3):648-655. doi: 10.1016/j. jid.2018.10.012.

9. The European Medicines Agency - EMA. Caelyx Anexo I: Ficha técnica o resumen de las características del producto 1. Agencia Europea de Medicamentos. EMA, 2006. Disponible en: https:// www.ema.europa.eu/en/medicines/human/paediatric-investigation-plans/etanercept

10. Gruss A, Torres S, Salisbury J, Quintana E, Curbelo P. Primer caso en Uruguay de tuberculosis asociada a terapia con agentes biológicos: reporte de un caso clínico. Rev Méd Urug. 2012:28(3),205-208. Disponible en: http://www. scielo.edu.uy/scielo.php?script $=$ sci_arttext\&pi$\mathrm{d}=$ S1688-03902012000300007\&lng=es.

11. Strady C, Brochot P, Ainine K, Jegou J, Remy G, Eschard JP, Jaussaud R. Tuberculose lors d'un traitement par agents inhibiteurs du TNF alpha. P R Health. Presse Med. 2006;35(11),1765-1772. doi: 10.1016/j.lpm.2006.09.019

12. Sharma SK, Mohan A, Sharma A. Challenges in the diagnosis \& treatment of miliary tuberculosis. Indian J Med Res. 2012:135(5),703-730. PMID: 22771605; PMCID: PMC3401706.

13. Martínez M, Martínez E, Pacheco O, Pérez S, López M, Pérez F, López M. Protocolo de Vigilancia en Salud Pública TUBERCULOSIS. 2016. Fecha de consulta: 11 de Julio 2020. Disponible en: http://www.clinicamedihelp.com/documentos/protocolos/PRO\%20Tuberculosis.pdf 